\title{
Switch-specific and general preparation map onto different ERP components in a task-switching paradigm
}

Frini Karayanidis, Alexander Provost, Scott Brown, Bryan Paton and Andrew Heathcote

Functional Neuroimaging Laboratory, School of Psychology and Centre for Brain and Mental Health Research, University of Newcastle, Callaghan NSW 2308 AUSTRALIA

\section{Corresponding author:}

Dr Frini Karayanidis

Email: frini.karayanidis@newcastle.edu.au

Telephone: +612 49215457

Fax: +612 49216980

Running head: Switch-specific and general preparation in cued task-switching

No.Tables: 0

No.Figures: 3

Acknowledgements

We thank Rebecca Nicholson for access to the original data, Damien Mannion and Tony Kemp for programming support, Ross Fulham for access to EEGDisplay software and Pat Michie for discussions related to this work. This work was supported by funding from the University of Newcastle Research Grants Committee and approved by the University of Newcastle Human Research Ethics Committee. 


\begin{abstract}
We examined whether the cue-locked centroparietal positivity is associated with switch-specific or general preparation processes. If this positivity (300-400ms) indexes switch-specific preparation, faster switch trials associated with smaller RT switch cost should have a larger positivity as compared to slower switch trials, but no such association should be evident for repeat trials. We extracted ERP waveforms corresponding to semi-deciles of each participant's RT distribution (i.e., fastest to slowest $5 \%$ of trials) for switch and repeat conditions. Consistent with a switch-specific preparation process, centroparietal positivity amplitude was linked to slower RT and larger RT switch cost for switch but not repeat trials. A later pre-target negativity (500-600ms) was inversely correlated with RT for both switch and repeat trials, consistent with a general anticipatory preparation processes.
\end{abstract}


Task-switching paradigms require shifting between two or more tasks using an alternating task sequence (i.e., alternating runs) or random sequences of task or transition cues (i.e., cued trials). Studies have consistently shown that response time (RT) is longer on trials that require a switch as compared to a repeat in task (e.g., Rogers \& Monsell, 1995; Allport, Hsieh \& Styles, 1994). In cued trials paradigms, this RT switch cost reduces as the interval between an informative cue and stimulus onset (cue-stimulus interval: CSI) increases (e.g., Meiran, 2000) - suggesting the activation of control processes associated with task-set reconfiguration, e.g., activation of task goal and category-response rules relevant to the cued task (Rogers \& Monsell, 1995; Rubinstein, Meyer \& Evans, 2001; Meiran, 2000) or activation of a task mediator cue (Logan \& Schneider, 2006). We will use the term switch-specific preparation to refer to these processes. For a fixed CSI, RT switch cost also reduces with increasing response-stimulus interval (RSI), which has been attributed to passive processes associated with dissipation of the previously active task-set (Allport et al., 1994). Cued trials paradigms allow investigation of switch-specific processes by manipulating CSI, with fixed values of RSI controling for passive dissipation processes. Even at long CSIs and RSIs, a residual RT switch cost remains, suggesting either that switch-specific preparation can't be completed before stimulus onset (Rogers \& Monsell, 1995) or that the stimulus triggers processes that interfere with applying the recently activated task-set (Meiran, 2000).

In cued trials paradigms, event-related potential (ERP) waveforms time-locked to cue onset consistently show a large centroparietal positive shift for switch as compared with repeat trials (e.g. Keiffaber \& Hetrick, 2005; Minuissi, Marzi \& Nobre, 2005; Nicholson, Karayanidis, Poboka, Heathcote \& Michie, 2005; Nicholson, Karayanidis, Bumak, Poboka \& Michie, 2006a; Rushworth, Passingham \& Nobre, 2005). This cue-locked differential switch-positivity emerges as early as 200ms post-cue. With long CSIs, this differential switch-positivity peaks and often dissipates prior to stimulus onset, whereas with short CSIs it peaks after stimulus onset. Recent studies have found that the amplitude of the switchpositivity is related to behavioral indices of preparation (Kieffaber \& Hetrick, 2005; Lavric, Mizon, \& Monsell, 2008; but see Swainson, Jackson \& Jackson, 2006). Using temporal principal components analysis (PCA), Lavric et al. (2008) extracted a late slow parietal-positivity / anterior negativity component that maps onto the switch-positivity. This component was negatively correlated with a performance index of switch cost, supporting the argument that the switch-positivity is associated with preparation to switch task. Based on the assumption that fast RT responses are associated with greater preparation than slow RT responses (de Jong, 2000; Nieuwenhuis \& Monsell, 2002), Lavric et al. averaged cue-locked ERPs separately for the fastest and the slowest third (terciles) of RT responses for each individual. A significant differential switch-positivity was evident for fast but not slow RT terciles, supporting a relationship between the amplitude of cue-locked switch positivity and switch-specific preparation for an impending switch trial. Goffaux et al. (2006) showed that the cue-locked posterior positivity was larger for switch than 
repeat trials equated for RT, indicating that it likely reflects differences in preparation for the upcoming trial, rather than generalized speed differences.

There is increasing evidence that a number of preparation-related ERP components are superimposed within the cue-target interval. In Keiffaber and Hetrick (2005), spatiotemporal PCA resulted in three components within the latency range of the switch-positivity mapped to different aspects of preparation. Partially informative cues indicating a switch away from the current task but not specifying the identity of the upcoming task elicited an early cue-locked differential switch-positivity, whereas fully informative cues also elicited a later switch-positivity within the CSI (Karayanidis, Mansfield, Galloway, Smith, Provost, \& Heathcote, 2009; Nicholson, Karayanidis, Davies \& Michie, 2006b). In cue-locked waveforms, a positivity can often be seen for both switch and repeat trials, although it is larger for switch trials (Goffaux, Phillips, Sinai \& Pushkar, 2006; Kieffaber \& Hetrick, 2005; Nicholson et al., 2005). Informative cues that validly signal either a switch or a repeat in task elicit an early posterior positivity relative to non-informative cues (that signal an upcoming target, but not the task), whereas informative switch cues also showed a later posterior positivity relative to informative repeat cues (Jamadar, Michie \& Karayanidis, 2010). Lavric et al. (2008) reported PCA components that temporally dissociated an earlier P3b from a later switch-specific positivity.

A late frontal negative component has also been identified, more frequently with a common average reference (e.g., Astle, Jackson \& Swainson, 2008; Mueller, Swainson \& Jackson 2007, 2009), that appears to be associated with response set preparation. In addition, with CSI of around 600-1000ms, cue-locked ERP waveforms often show a pre-target negativity emerging for both switch and repeat trials, although it is usually larger for repeat trials (e.g., Nicholson et al., 2005). This pre-target negativity is likely to reflect anticipatory attention (i.e., stimulus-preceding negativity, SPN) and/or response readiness (i.e., contingent negative variation, CNV), which may also vary across the RT distribution and possibly affect RT switch cost (see Karayanidis, Jamadar, Ruge, Phillips, Heathcote \& Forstmann, submitted, for a recent review).

At a behavioral level, there is increasing evidence that RT switch cost does not always reflect an increase in switch trial RT relative to a constant repeat trial RT baseline, but may also reflect variation in repeat trial RT. For example, in a study of sequence effects in task-switching, Koch and Phillips (2005) found that RT switch cost was eliminated on trials preceded by a no-go trial. However, this effect was largely due to an increase in repeat trial RT rather than a reduction in switch trial RT. Jamadar, Michie and Karayanidis (2010) demonstrated that this effect is due to reduced response readiness on the repeat trials. Altmann (2004) posits a general preparatory process that reduces RT for both switch and repeat trials as CSI increases. This anticipatory preparation process is argued to reflect a primary process of memory retention that is strongest for repeat trials and maintains the previously active task-set. Altmann argues that 
any preparation for switch trials may or may not be subsumed under this general preparation process. A central aim of our study is to examine preparation effects separately on both switch and repeat trials. In particular, we examine whether variation over the range of the RT distribution affects the amplitude of the cue-locked positivity and the pre-target negativity for both switch and repeat trials.

Variation in ERP Components with RT

ERP component amplitude varies in the range of $1-20 \mu \mathrm{V}$, whereas EEG ranges from -100 to +100 $\mu \mathrm{V}$ (Regan, 1989; Handy, 2004). The most common method to extract ERPs involves time-locked signal averaging over many trials, so that large EEG “noise” fluctuations average to zero and the embedded ERP signal is extracted. Depending on the nature of the ERP component, the number of trials needed to obtain a satisfactory signal-to-noise ratio can vary considerably. Long-latency components associated with higher order cognitive processes, like the differential switch-positivity, often manifest as small variations between experimental conditions. These difference waveforms tend to produce relatively small ERP components (approx. 1-5 $\mu \mathrm{V}$ ) with comparatively low signal-to-noise ratio, and so requirie averaging over a large number of experimental trials.

Signal averaging relies on the assumption that the ERP signal remains constant across repeated presentations of the stimulus. However, there is ample evidence that this assumption is not always valid. Successive presentations of the stimulus may result in systematic changes in the ERP component over the course of the testing session, and in some instances can result in important component information being “averaged out” with noise (Spencer, 2004). In the context of most research questions addressed by ERP studies, violation of this assumption does not have a critical impact on the validity of the conclusions. In other contexts, however, variation in behaviour and ERP components over time is central to the research focus (e.g., Woestenberg, Verbaten, Van Hees \& Slangen, 1983; Lorist, Klein, Nieuwenhuis, De Jong, Mulder, \& Meijman, 2000; Karayanidis Robaey, Bourassa, De Koning, Geoffroy, \& Pelletier, 2000; Shelley, Ward, Michie, Andrews, Mitchell, Catts, et al., 1990).

There is emerging evidence that switch-related preparation varies as a function of RT. As mentioned earlier, Lavric et al. (2008) found that ERPs derived by averaging over trials with broadly similar RTs (i.e., the fastest and slowest thirds of the RT distribution) differ in the morphology of cuelocked components associated with advance preparation. Here we focus on the variation of ERP components with trial-to-trial variations in RT. We took a different approach to Lavric's, using a modified version of Woestenburg et al.'s (1983) orthogonal polynomial trend analysis (OPTA) technique (see the Methods section for details). In this approach, RTs for each trial are used as a covariate in a regression model of the observed EEG signals. The regression model can then be used to examine ERP components for any single observed RT value, enabling a fine-grained analysis of component changes with RT. The 
signal-to-noise issue is addressed because all trails contribute to the regression model. As changes in RT switch cost over the RT distribution (de Jong, 2000; Poboka et al., 2005) likely reflect, at least in part, variation in anticipatory preparation, the amplitude of the differential switch-positivity was expected to vary systematically across the RT distribution.

We applied the OPTA analysis to data previously examined with conventional ERP averaging by Nicholson et al. (2005) and with RT distributional analyses by Poboka et al. (2005). In the current paper, we report results only from two conditions with a 600ms CSI, as these conditions showed a substantial reduction in switch cost relative to a short CSI condition $(150 \mathrm{~ms})$ and a plateau in residual switch cost relative to a longer CSI condition (1050ms). Analyses were targeted to two ERP components evident in cue-locked waveforms: the centroparietal positivity over 300-400ms and the frontocentral pre-target negativity over $500-600 \mathrm{~ms}$, both of which contribute to the differential switch-positivity. Variation in RT as a result of general preparation following cue onset would be expected to be evident on both switch and repeat trial ERPs, whereas variation associated with switch-specific preparation would be expected to occur for switch trials only. Based on evidence that the cue-locked positivity is related to processes associated with switch-specific preparation, faster RT trials that are associated with smaller RT switch cost were expected to show larger cue-locked positivity than slower RT trials after switch but not repeat cues. As the pre-target negativity is likely to be associated with general preparation processes such as anticipatory attention and response readiness, faster RT trials were expected to be associated with larger pre-target negativity than slow RT trials after both repeat and switch cues.

\section{Method}

\section{Participants}

Twenty-four students from the University of Newcastle (18 to 30 years of age, mean $=22.2$ years, 15 female) enrolled in an introductory psychology course completed this study for course credit.

\section{Paradigm $^{1}$}

A rectangular box was divided into four equal quadrants and continuously displayed on a monitor at a viewing distance of $90 \mathrm{~cm}$. Participants alternated randomly between two tasks. The letter classification task involved classifying a letter as consonant (G, K, M, R) or vowel (A, E, I, U). The number classification task involved classifying the digit as odd $(3,5,7,9)$ or even $(2,4,6,8)$. Responses were mapped to the left and right hand and response hand mapping was counterbalanced across participants. The letter task was

\footnotetext{
${ }^{1}$ See Nicholson et al. (2005) for full details on the paradigm.
} 
assigned to the top two quadrants for half of the participants and to the right two quadrants for the other half in order to counterbalance the mapping between eye shift (vertical or horizontal) and trial type (switch or repeat). Stimuli were pairs of Times New Roman font characters. One of the characters was selected from the currently active task set (e.g. letters for the letter task). On one third of trials, the second character was a non-alphanumeric character (i.e. \#, \%, ?, *) that was not mapped to any response (neutral pairing). On the remaining trials, the task irrelevant character was selected from the other task (i.e., number for letter task) with half of these trials involving a congruent pairing (relevant and irrelevant characters mapped to the same response) and the other half involving an incongruent pairing (mapped to opposite responses). Character position (e.g. E\#, \#E) varied randomly across trials. Each trial began with a cue indicating the quadrant in which the next stimulus would be presented. The cue was a highlight of the line defining that quadrant (from gray to white) and remained on throughout the duration of the stimulus presentation. The stimulus remained on screen until a response was emitted or 5000ms elapsed. The next cue appeared in one of the two adjacent quadrants (horizontal or vertical) and its position defined a switch or repeat trial. The first four trials of every block, incorrect response trials, the trial following an incorrect response and any trial with a response outside 200-2000ms post-stimulus were not included in data analysis.

RSI and CSI were manipulated across different blocks resulting in a total of six timing conditions presented in counterbalanced order. Only two timing conditions with CSI of 600ms (blocked RSI of 750ms and 1200ms) will be presented in this paper (3x100 trials per RSI). These two timing conditions were selected because they resulted in a large clear switch positivity that fully resolved within the CSI (Nicholson et al., 2005).

\section{EEG Recording and Data Analysis}

EEG was continuously sampled from 12 scalp electrodes according to the $10 / 20$ system (Fz, $\mathrm{Cz}, \mathrm{Pz}$, F3, C3, P3, T5, F4, C4, P4, T6, Oz) with linked mastoids reference (500 Hz/channel; NeuroScan Acquire; GrassNeurodata system (Model 12); bandpass: 0.01-30 Hz, -6 dB down). Vertical and horizontal electrooculogram was recorded bipolarly from electrodes attached to the supraorbital and infraorbital ridges of the left eye and the outer canthi of each eye, respectively. EEG was corrected for eyeblink artifact (Semlitsch, Anderer, Schuster, \& Presslich, 1986) and sections with movement artifact or channel saturation were excluded from further analyses. Cue-locked EEG epochs for trials associated with a correct response were extracted over an interval spanning 200ms before and $1200 \mathrm{~ms}$ after cue onset (baseline +/-50 ms).

\section{OPTA Analysis}

In order to examine variation in differential switch-positivity as a function of changes in RT switch cost over the RT distribution, we applied a revised version of the OPTA technique developed by Woestenburg et al. (1983). OPTA uses a polynomial regression model of EEG components in the frequency 
domain to estimate how that component changes with a covariate. The process involved transforming EEG data into the frequency domain and applying an orthogonal polynomial regression equation, using RT as the only covariate, to each component of the frequency profile. Polynomial coefficients up to and including the $5^{\text {th }}$ order term were used in component estimation only when the regression analysis suggested that they explained a significant proportion of variance.

Orthogonal polynomial trend analysis has been used previously to model a variety of time-varying neurophysiological phenomena (de Koning, Woestenburg \& Elton, 2001; Karayanidis et al., 2000; Kenemans et al., 1992; van der Lubbe \& Woestenburg, 1997; 1999; 2000; Woestenburg et al., 1983). For example, in a habituation study, Woestenburg et al. used OPTA to examine changes in ERPs as a function of trial. OPTA allows the estimation of ERP components on an individual trial-by-trial level, whereas conventional techniques require averaging over a large number of trials to improve signal to noise ratio. Woestenburg et al. showed that OPTA can achieve about a tenfold improvement in the signal-to-noise ratio when ERP components change with some covariate, and that OPTA outperforms another quite sophisticated averaging technique (Wiener filtering).This OPTA procedure used here differs from the original algorithm implemented by Woestenburg et al. in that it uses RT as a covariate. We applied OPTA on epoched EEG data using software written in MATLAB®. For each participant, RSI condition and trial type, cue-locked epochs associated with correct responses were ranked based on RT: for example, the fastest RT recorded by the participant for repeat trials in a particular RSI condition was assigned the covariate value " 1 ”, the next fastest " 2 ", and so on. These covariates correspond to the order statistics of RT samples ${ }^{2}$. The EEG epochs were then transformed using a fast Fourier transform (FFT). A polynomial regression equation - similar to a trend analysis in a conventional ANOVA - was applied at each frequency, using the order statistics as the covariate and polynomial terms up to the fifth order. The proportion of analyses on which the highest-order polynomial coefficient $\left(5^{\text {th }}\right)$ explained significant variance was smaller than the Type I error rate in both conditions (4.2\% for repeat, $3.7 \%$ for switch) indicating that analysis of even higher-order terms (e.g., $6^{\text {th }}, 7^{\text {th }}$ etc) was not necessary.

After removing terms that failed to explain significant variance, the fitted polynomial functions were used to generate predicted waveforms in the frequency domain, corresponding to each trial. This was achieved by using the polynomial equation at each frequency to generate predicted power values for order statistics corresponding to the RT value of each trial for each participant. Finally, these RT-ranked predicted waveforms were transformed back into the time domain using the inverse FFT. This resulted in an RT

\footnotetext{
${ }^{2}$ We also conducted the analyses using RT magnitude rather than order statistics as the covariate. This analysis required a further modification of the original OPTA analysis, as it necessitated using orthogonal polynomial coefficients for unequally spaced covariates. The conclusions obtained with both types of covariate were the same.
} 
ranked waveform for each trial for each participant. For each trial type, group average waveforms corresponding to $5^{\text {th }}, 15^{\text {th }}$ etc up to the $95^{\text {th }}$ percentile of the RT distribution were created by averaging across participants and these were used to depict the variation in cue-locked ERP waveforms over the RT distribution. For statistical analyses, RT ranked single-trial waveforms were averaged within each of the 20 semi-decile intervals for each participant (4-7 trials per semi-decile). This procedure resulted in 20 cuelocked waveforms per individual for each condition and trial type which represent the averaged waveform corresponding to RT percentile values incrementing by $5 \%$, starting at the $2.5^{\text {th }}$ percentile.

\section{Data Analysis}

The mean RT for each semi-decile interval was analysed using a 2 RSI condition (750, 1200ms) x 2 trial type (repeat, switch) x 20 RT intervals repeated measures GLM in SPSS. Since we were specifically interested in examining variation in cue-locked waveforms as a function of RT, we targeted analyses at frontal, central and parietal sites over the midline and right scalp, as initial visual inspection of the averaged data indicated that these locations showed the largest effects of decile. Based on the conventionallyaveraged data presented by Nicholson et al. (2005), we defined two mean amplitude windows to target the cue-locked positivity (300-400ms) and the pre-target negativity (500-600ms). ERPs were analysed using a 2 RSI x 2 trial type x 20 RT intervals x 3 coronal (frontal, central, parietal) x 2 lateral (midline, right) repeated measures analyses at each of the two mean amplitude windows. Planned linear and quadratic polynomial comparisons are reported in all analyses of the RT interval factor. For both behavioral and ERP analyses, degrees of freedom for factors with more than two levels were adjusted using Greenhouse-Geisser correction for the violation of the assumption of sphericity (Vasey \& Thayer, 1987).

\section{Results}

Figure 1 shows RT and mean amplitude measures from cue-locked waveforms for switch and repeat trials at each RT percentile interval. Note that each value represents the mean across a 5\% range, so that the first value corresponds to the mean value under $5 \%$ (i.e., an average corresponding to the mid-point of the rank, $2.5^{\text {th }}$ percentile), the second to the mean value between 5-10\% etc. As shown in Figure 1 (left), RT was significantly faster for repeat than switch trials $(F(1,23)=60.3, p<.001)$. RT increased significantly across percentiles $(F(19,437)=328, p<.001)$ with significant linear $(F(1,23)=340, p<.001)$ and quadratic $(\mathrm{F}(1,23)=254, \mathrm{p}<.001)$ trends. More importantly, RT switch cost was affected by RT percentile rank $(\mathrm{F}(19,437)=36.8, \mathrm{p}<.001)$ increasing linearly from $26 \mathrm{~ms}$ at the first percentile interval to $157 \mathrm{~ms}$ at the last percentile interval $(\mathrm{F}(1,23)=96.2, \mathrm{p}<.001)$. Residual switch cost was significant even for the fastest $5 \%$ of RT responses $(\mathrm{F}(1,23)=41.6, \mathrm{p}<.001)$. There was no significant interaction between RSI condition and RT rank. 
Figure 2A shows cue-locked ERP difference waveforms derived by subtracting the repeat trial from the switch trial ERP waveform for corresponding percentile values. Overall, these difference waveforms show a typical centroparietally maximal differential switch positivity, emerging earliest centrally and extending beyond stimulus onset parietally. The variation in the amplitude of the switch-positivity across the RT distribution is striking, with a much larger switch-positivity for fast as compared to slow RT trials. Note that fast RT trials are associated with a prolonged centroparietal switch-positivity throughout most of the CSI, whereas slow RT trials show little differentiation between switch and repeat trials beyond 450ms. In order to examine whether these effects are due to RT-related variation on switch and/or repeat trial ERPs, Figure 2B depicts ERP waveforms for repeat and switch trials separately. Both switch and repeat trial ERPs showed a centroparietally maximal positivity over 200-400ms. This positivity was much larger for switch trials, especially over 300-400ms, reflecting the differential switch positivity seen in many previous taskswitching studies. This was followed by a fronto-central pre-target negativity emerging around 200ms before target onset that tended to be larger for repeat trials. Two mean amplitude windows targeted the parietal positivity (300-400ms) and the pre-target negativity (500-600ms) where the effects of decile were most pronounced. Although both ERP mean amplitude windows showed a significant effect of RSI condition (300-400: $\mathrm{F}(1,23)=18.9, \mathrm{p}<.001,500-600: \mathrm{F}(1,23)=8.0, \mathrm{p}<.009)$, reflecting a greater positive drift in the entire waveform for the long as compared to short RSI, this factor did not interact with RT percentile rank in either 300-400ms or 500-600ms windows.

Switch trials had more positive values than repeat trials in both epochs (Figure 2A), reflecting a larger centroparietal positivity over $300-400 \mathrm{~ms}$ followed by a smaller pre-target negativity over 500-600ms (300-400: $F(1,23)=41, \mathrm{p}<.001,500-600: \mathrm{F}(1,23)=6.5, \mathrm{p}<.018)$. The OPTA procedure resulted in ERP waveforms that varied quite systematically from fast to slow RT deciles with little variation in the interval preceding cue onset. Note that the effect of RT percentile on the amplitude of the cue-locked waveforms varied across the CSI and for switch and repeat cues (Figure 2B). For repeat cues, the effect of RT variation was restricted to the amplitude of the stimulus-preceding negativity with faster RT percentile intervals showing larger negativity. Switch cues showed a similar though smaller effect of RT variation on the stimulus-preceding negativity. In addition, switch cues showed the opposite effect on the amplitude of the centroparietal positivity with faster RT percentiles showing a larger positivity. While the RT percentile factor resulted in significant main effects and/or interactions with trial in both epochs, these did not reflect a uniform effect of percentile across both trial types, but rather a selective pattern of effects for repeat and switch trials on different ERP components. Figure 1 (right) shows mean amplitude of repeat and switch ERPs at each RT percentile for each mean amplitude window.

\section{Centroparietal positivity (300-400ms)}


The centroparietal positivity (300-400ms) produced a significant RT interval x trial type interaction $(\mathrm{F}(19,437)=10.1, \mathrm{p}<.001)$. Repeat trials showed a small and sustained positivity of around $4 \mu \mathrm{V}$ parietally that did not vary significantly with increasing RT percentile rank (Figure 2B; F<1). In contrast, switch trials showed a larger positivity than repeat trials ( $7.5 \mu \mathrm{V}$ parietally) and the amplitude of this positivity varied with RT percentile rank. This positivity varied as a function of RT interval $(F(19,437)=10.1, \mathrm{p}<.001)$ showing a significant linear decline with increasing $\mathrm{RT}(\mathrm{F}(1,23)=14.2, \mathrm{p}<.001)$. So, fast switch responses that were associated with small RT switch cost (Figure 1 left) showed a larger overall positivity and a larger differential switch positivity as compared to slow switch responses that were associated with large RT switch cost. Note that, although the cue-locked positivity difference between switch and repeat trials reduced with increasing RT, the positivity was still significantly larger for switch as compared to repeat trials even at the slowest $5 \%$ of the RT distribution (i.e., $95^{\text {th }}$ percentile; $F(1,23)=20, p<.001$; see Figure 2C).

We examined inter-individual variability in RT and cue-locked positivity by analysing correlations between mean RT and mean amplitude of the cue-locked positivity over 300-400ms averaged over RT percentile interval and RSI condition using one-tailed Pearson correlations. RT switch cost showed a very high and significant correlation with switch trial RT ( $r=.821, \mathrm{p}<.001)$ and a smaller correlation with repeat trial RT ( $r=.456, \mathrm{p}<.013$ ), indicating that individual variation in RT switch cost was more strongly related to differences in switch rather than repeat trial RT. As expected, there was no significant correlation between repeat trial RT and cue-locked positivity for repeat trials ( $\mathrm{p}>$.17). For switch trials, mean RT was negatively correlated with cue-locked positivity amplitude over the right parietal scalp (P4: $r=-.405, \mathrm{p}<.025$ ), as well as with the amplitude of the differential switch positivity over central and parietal scalp (Pz, C4, P4: -.409 < $r<-.480, \mathrm{p}=.024$ to .009). These findings indicate that individuals with faster switch trial RT tended to elicit a larger positivity for switch trials and a larger switch-repeat differential positivity as well as a smaller RT switch cost. Together with the semi-decile analyses above, these results show effects of both intra- and inter-individual variability in cue-locked positivity affects performance, such that faster RT was associated with smaller RT switch cost, as well as with larger cue-locked differential positivity for switch as compared to repeat trials.

\section{Pre-target negativity (500-600ms)}

The effect of RT percentile rank was even more pronounced on the pre-target negativity which showed a significant linear reduction with increasing $\mathrm{RT}(\mathrm{F}(19,437)=22.3, \mathrm{p}<.001$; linear contrast: $\mathrm{F}(1,23)=27.47, \mathrm{p}<.001)$. The effect of percentile was more pronounced centrally $(\mathrm{F}(38,874)=9.8, \mathrm{p}<.001)$ and over the midline $(\mathrm{F}(19,437)=9.3, \mathrm{p}<.001)$. Although the reduction in negativity with increasing RT percentile was evident for both switch and repeat trials, the effect was more pronounced in the latter (Figure 
1; interval $\mathrm{x}$ type: $\mathrm{F}(19,437)=3.9, \mathrm{p}<.035$; interval $\mathrm{x}$ type $\mathrm{x}$ coronal: $\mathrm{F}(38,874)=4.5 \mathrm{p}<.008)$. Overall, repeat trials reduced by $1.8 \mu \mathrm{V}$ and switch trials by $1.2 \mu \mathrm{V}$ from the fastest to the slowest $\mathrm{RT}$ percentile. Repeat trials showed a linear reduction in negativity with increasing RT percentile $(F(1,23)=34.9, \mathrm{p}<.001)$, whereas switch trials showed both linear and quadratic trend $(F(1,23)=8.9, \mathrm{p}<.007 ; \mathrm{F}(1,23)=8.4, \mathrm{p}<.008)$ reflecting a plateau during the second half of the RT distribution. So, for both repeat and switch trials, a larger pre-target negativity was associated with a faster response to the upcoming target. The pre-target negativity amplitude difference between switch and repeat trials reduced across the RT distribution (Figure 1, right). At central sites, where the pre-target negativity was largest, switch and repeat trials did not differ significantly beyond the $75^{\text {th }}$ RT percentile ( $>$ >.10).

We examined inter-individual variability in RT and pre-target negativity by analysing correlations between mean RT and mean amplitude over 500-600ms averaged over RT percentile interval and RSI condition using one-tailed Pearson correlations. Pre-target negativity amplitude was primarily related to RT for repeat trials. Specifically, on repeat trials, a large pre-target negativity and a large switch-repeat difference were associated with faster RT. These correlations were stronger centroparietally (repeat negativity \& repeat RT: $r=.364-.403, p=.04-.026$; switch-repeat difference and repeat RT over right scalp: $r=-.437-.461, p=.012-.016)$. So, inter-individual variability in pre-target negativity was associated with variability on repeat trial RT.

\section{Analyses of cue-locked waveforms for switch and repeat trials with equivalent $R T$}

Task switching paradigms produce a significant RT switch cost in most experimental conditions. So ERP waveforms for switch and repeat trials differ not only in trial type but also in mean RT. Therefore, cuelocked ERP differences between switch and repeat trials could be confounded by non-specific factors affecting overall RT rather than processes specifically associated with anticipatory preparation for an impending change in task-set. The OPTA technique provides a unique capability to compare cue-locked ERP waveforms for switch and repeat trials equated for RT within a very narrow band (see Goffaux et al., 2006, for a related approach). Within each condition, we identified RT percentile ranks around the middle of the distribution that had very similar mean RT for switch and repeat trials. Specifically, repeat trial RT from $50^{\text {th }}$ and $55^{\text {th }}$ percentile from short and long RSI conditions, respectively, was closely matched with switch trial RT from $35^{\text {th }}$ percentile in both RSI conditions (short RSI: 556 vs. 559ms; long RSI: 551 vs. 556ms, for repeat and switch respectively; trial type and RSI condition effects $\mathrm{F}<1$ ). These data were entered into a 2 condition x 2 trial type x 3 coronal x 2 lateral repeated measures ANOVA for each mean amplitude window. Over 300-400ms, the main effect of type was highly significant $(F(1,23)=36.6, p<.001)$, indicating a larger cue-locked positivity for switch than repeat trials matched for mean RT (Figure 2D). This difference between switch and repeat trials showed the same centroparietal midline pattern seen when 
averaging across all trials (type $\mathrm{x}$ coronal $\mathrm{x}$ lateral: $\mathrm{F}(2,46)=9.2, \mathrm{p}<.001)$. In contrast, the pre-target negativity (500-600ms) showed no main effect of trial type $(\mathrm{F}<1)$ and a significant trial $\mathrm{x}$ coronal interaction $(\mathrm{F}(2,46)=16.9, \mathrm{p}<.001)$, resulting largely from the slower resolution of the parietal positivity for switch trials.

\section{Comparing OPTA findings against conventional averaging}

A full methodological investigation of the benefit of the OPTA procedure applied in this study as compared to conventional averaging and other methods of small-trial ERP analyses is beyond the scope of the current paper (see Provost, Brown, Heathcote, Karayanidis, in preparation). For the aims of the current study, we examined whether we would be able to obtain the same findings with more conventional signal averaging. For this purpose, we processed the ERP epoched data in an identical manner, but rather than applying the OPTA technique, we simply averaged over the ERP epochs within each of the semi-deciles. As expected, these conventional averages showed much greater variability than the OPTA-derived waveforms (Figure 3). This was confirmed by signal-to-noise measures, which showed that mean amplitude in the 300-400ms window derived from OPTA waveforms were associated with 2.5 times greater signalnoise ratio than conventional averaging. In particular, a test of all 480 conditions gave an $\mathrm{F}(479,11017)=20.7$ for OPTA vs. 8.4 for conventional averaging. When we ran the mean amplitude analyses on the conventional averages over the 20 semi-deciles, the pattern of results was largely the same as for the OPTA analysis, although the associated F-values were smaller and sometimes failed to reach statistical significance.

\section{Discussion}

In this paper we applied an OPTA analysis (Woestenburg et al., 1983), using individual trial RTs as a covariate, to examine cue-locked ERP waveforms in a task-switching paradigm. Our aim was to determine whether variability in anticipatory preparation as indexed by variation in RT switch cost across the RT distribution is associated with variation in the cue-locked waveforms exclusively for switch trials or for both switch and repeat trials. At a relatively long preparation interval of 600ms, increasing RT was associated with larger RT switch cost (de Jong, 2000, Nieuwenhuis \& Monsell, 2002), with as much as a 5-fold increase in RT switch cost from the fastest to the slowest RT semi-deciles. We used the OPTA technique to derive ERP waveforms for twenty RT percentile rank values spread across the entire RT distribution. ERP waveforms showed a stable profile across RT percentile rank with a much higher signalnoise ratio than could be achieved by conventional averaging. Variability associated with RT percentile 
very selectively affected specific components of the ERP waveform differentially for switch and repeat trials.

A cue-locked centroparietal positivity emerged around 200ms, peaked around 350ms and was larger for switch than repeat trials (Nicholson et al., 2005). OPTA analyses showed that, for repeat trials, this positivity did not vary in amplitude across the range of the RT distribution. However, for switch trials, the cue-locked positivity was significantly larger for fast as compared to slow RT trials. Therefore, the fastest end of the RT distribution, which showed the smallest RT switch cost and the most prepared responses, was associated with the largest centroparietal positivity for switch trials. The slowest end of the RT distribution, which displayed the largest RT switch cost and represented the least prepared switch responses, was associated with the smallest positivity for switch trials. As there was no significant variation in positivity for repeat trials over this latency range, the reduction in differential switch-positivity with increasing RT switch cost can be attributed to the variation in switch trial positivity. This is consistent with the finding that inter-individual variability in switch trial, but not repeat trial, RT was negatively correlated with the amplitude of the cue-locked positivity for switch trials and with the amplitude of the differential switch-positivity.

Despite a progressive reduction in differential-switch positivity over the RT range, the cue-locked difference between switch and repeat trials for the slowest $5 \%$ of responses was still significant. In addition, significant differences in cue-locked ERPs between switch and repeat trials remained even when the RT difference that is normally obtained between these trials was eliminated (see also Goffaux et al., 2006). Taken together, the findings that a) the most prepared responses show the largest switch-repeat differentiation (fastest 5\%), b) the amplitude of this positivity differs significantly between switch and repeat trials even at the lowest point of amplitude differentiation (slowest 5\%), and c) the difference in positivity is evident even when repeat and switch trials are matched on RT, suggest that the differential switch-positivity represents a component elicited specifically on switch trials. This component is superimposed on a more generic positivity that is also elicited for repeat trials, and its amplitude is associated with the amount of switch-specific preparation. This conclusion is also supported by the finding that the positivity for repeat trials showed little change over RT semi-deciles, despite the fact that repeat and switch RT varied over largely overlapping ranges (380-1250ms for repeats, 405-1405ms for switches). These findings suggest that cue-locked ERPs consist of a positivity for repeat and switch trials over 200400ms whose amplitude is not associated with variation in RT and a superimposed positivity, especially over the later part of that interval, that occurs exclusively for switch trials and whose amplitude is inversely related to RT and RT switch cost (see also Lavric et al. 2008). These findings are consistent with at least 
two preparatory processes: one that is common to both switch and repeat trials and another that, at least in the present context, is specifically-elicited by switch cues.

The positivity associated with both switch and repeat trials is consistent with the larger positivity found for repeat trials in a mixed-task block as compared to trials in a single-task block (i.e., mixing positivity; Goffaux et al., 2006; Jost, Mayr \& Rosler, 2008; Wylie et al., 2009; Ruge, Stoet \& Naumann, 2006) and for informative repeat cues compared to non-informative cues (Jamadar et al., 2010). Wylie et al. (2009) showed that this mixing positivity was associated with LAURA source activation in parietal areas. Jamadar, Hughes, Fulham, Michie and Karayanidis (2010) found that variation in the amplitude of this mixing positivity was associated with fMRI contrast activation in dorsolateral prefrontal cortex and posterior cingulate. The switch-specific positivity is compatible with a process associated with anticipatory preparation that, at least with the current task parameters, is only engaged on switch trials ${ }^{3}$. The gradual variation in switch-positivity amplitude and its association with RT switch cost suggests that the switchspecific reconfiguration process varies in efficiency across trials resulting in intra-individual variability in RT switch cost. The switch-specific positivity we observed is compatible in latency with the switch-specific activation shown in Wylie et al. Figure 5B over frontal sources. Lavric et al. (2008) found greater frontal (500-600ms) followed by later posterior (600-800ms) LORETA activity for the switch - repeat contrast. Jamadar et al (in press) showed that the switch-repeat positivity was associated with fMRI contrast activation in posterior parietal cortex. While the latency and, to some degree, the morphology of these switch-positivities differ across these studies, this may be due to differences in EEG reference settings, complexity of the experimental paradigm and amount of training.

Although the positive relationship between RT and RT switch cost is generally compatible with de Jong's (2000) intention-activation model's stipulation of a mixture of both prepared and unprepared trials, the gradual variation in cue-locked switch positivity amplitude with increasing RT switch cost is not easily reconciled with an all-or-none process (see also Brown, Lehmann \& Poboka, 2006). Furthermore, we found a significant RT switch cost even for the fastest $5 \%$ of responses. This again argues against a binary division between fully prepared vs. unprepared states, and suggests either that anticipatory reconfiguration cannot be fully completed until after stimulus onset (Rogers \& Monsell, 1995) or that S-R interference processes activated by the target (Allport \& Wylie, 2000) affect RT even for fully prepared trials. These findings suggest that switch-specific preparation may involve a gradual process or a set of sub-processes that may be partly or fully completed within the cue-target interval. This conclusion is compatible with

\footnotetext{
${ }^{3}$ Alternatively, as suggested by an anonymous reviewer, this switch-positivity may represent the different outcome of a process that is equally activated for both switch and repeat trials. As we cannot differentiate between these alternatives here, we do not discuss this alternative further.
} 
other data suggesting a multi-component process of advance preparation (Jamadar et al., 2010b; Karayanidis et al., 2009; Nicholson et al., 2006b; Swainson, Jackson \& Jackson, 2006) which are differentially activated depending on task parameters and strategy (see Karayanidis et al., submitted).

In contrast to the finding that the amplitude of the cue-locked positivity varies across the RT distribution for switch trials only, the amplitude of the pre-target negativity was related to RT for both switch and repeat trials. The pre-target negativity was larger for repeat as compared to switch trials and for fast as compared to slow responses on both repeat and switch trials. Fast switch trials were associated with a larger positivity as well as a larger pre-target negativity as compared to slow switch trials. This indicates that RT had opposite effects on the switch waveform in these two epochs (Figure 2B) and suggests that the effect of RT percentile on the pre-target negativity does not simply reflect a carry-over of the earlier effect on the switch positivity. The independence of cue-locked positivity and the pre-target negativity associations to RT is also supported by the fact that, although pre-target negativity amplitude varied across RT percentile for repeat trials, there was no variability in the cue-locked positivity for repeat trials. So, intra-subject variability showed that a larger pre-target negativity was associated with faster responding for both switch and repeat trials, whereas inter-subject variability showed that fast responders tended to show a larger pre-target negativity for repeat trials only. Note that eliminating RT differences between switch and repeat trials eliminated the difference in pre-target negativity amplitude between these trial types at central electrodes. The variation in pre-target negativity amplitude with RT is consistent with a more general arousal or attention-related process and suggests that the switch / repeat difference in pre-target negativity amplitude is more likely to be related to generic differences in preparation or task readiness.

In conclusion, application of the OPTA regression methodology to cue-locked waveforms provided evidence for both switch-specific and general anticipatory preparation processes within the CSI. What in the cue-locked difference waveforms appeared as a centroparietal switch-positivity spanning most of the CSI was, in fact, the result of differential modulation of two underlying components. The peak of the centroparietal differential switch-positivity mapped onto a cue-locked positivity that was inversely related to RT for switch trials only. The slow return to baseline of this differential switch-positivity mapped onto the pre-target negativity that was directly related to RT for both switch and repeat trials. These findings support multi-component models of anticipatory preparation and suggest that care needs to be taken when interpreting results based solely on difference waveform analyses. The cue-locked positivity and the pretarget negativity are compatible with the conceptualisation of advance preparation as a set of processes that may include central as well as modality-specific and/or task-specific components (Jennings \& van der Molen, 2005). The finding that the cue-locked positivity varied as a function of RT percentile only for switch trials suggests that, in the current context, this component reflects a switch-specific reconfiguration 
process. However, this does not preclude the possibility that, given the right task parameters (e.g., low repeat probability), advance reconfiguration may also be helpful on repeat trials. 


\section{References}

Allport, D. A., Styles, E. A., \& Hsieh, S. (1994). Shifting intentional set: Exploring the dynamic control of tasks. In C. Umilta \& M. Moscovitch (Eds.), Attention and Performance XV (pp. 421-452). Cambridge, MA: MIT Press.

Allport, D. A., \& Wylie, G. (2000). Task switching, stimulus-response bindings, and negative priming. In S. Monsell \& J. Driver (Eds.), Attention and Performance XVIII (pp. 35-70). Cambridge,MA: MIT Press.

Altmann, E.M (2004). The preparation effect in task switching: Carryover of SOA. Memory \& Cognition, 32, $153-163$

Brown, S.D., Lehmann, C., \& Poboka, D. (2006) A critical test of the failure-to-engage theory of taskswitching. Psychonomic Bulletin \& Review, 13, 152-159.

De Jong, R. (2000). An intention-activation account of residual switch costs. In S. Monsell \& J. Driver (Eds.), Attention and Performance XVIII (pp. 357-376). Cambridge, MA: MIT Press.

De Koning, D., Woestenburg, J., Elton, M. (2001). Visual Spatial Attention in Migraine Sufferers in Postictal and Interictal phases: An Event-Related Potential Study. Journal of Psychophysiology, 15, 22-34.

Goffaux, P., Phillips, N.A., Sinai, M., \& Pushkar, D. (2006). Behavioural and electrophysiological measures of task switching during single and mixed-task conditions. Biological Psychology, 72, $278-290$

Handy, T. (2004). Basic Principle of ERP Quantification. In T. Handy (Ed.), Event-Related potentials: A Methods handbook (pp.33-56). Cambridge, MA: MIT Press.

Jamadar, S., Hughes, M., Fulham, W.R., Michie, P.T. Karayanidis, F. (2010) The spatial and temporal dynamics of anticipatory preparation and response inhibition. Neuroimage, 51, 432-449 
Jamadar, S., Michie, P.T. Karayanidis, F. (2010) Sequence effects in cued task-switching modulate response preparedness and repetition priming processes. Psychophysiology, 47, 365-386

Jost, K., Mayr, U., \& Rosler, F. (2008). Is task switching nothing but cue priming? Evidence from ERPs. Cognitive, Affective \& Behavioral Neuroscience, 8, 74-84.

Karayanidis, F., Coltheart, M., Michie, P.T., \& Murphy, K. (2003). Electrophysiological correlates of anticipatory and post-stimulus components of task-switching. Psychophysiology, 40(3), 329-348.

Karayanidis, F., Robaey, P., Bourassa, M., De Koning, D., Geoffroy, G., \& Pelletier, G. (2000). ERP differences in visual attention processing between attention-deficit hyperactivity disorder and control boys in the absence of performance differences, Psychophysiology, 37, 319-333.

Karayanidis, F., Mansfield, E.L., Galloway, K.L., Smith, J., Provost, A., \& Heathcote,A. (2009). Anticipatory reconfiguration elicited by fully and partially informative cues that validly predict a switch in task. Cognitive, Affective \&Behavioural Neuroscience, 9 (2), 202-215

Kieffaber, P.D., \& Hetrick, W.P. (2005). Event-related potential correlates of task switching and switch costs. Psychophysiology, 42, 56-71.

Kenemans, J.L., Verbaten, M.N., Melis, C.J., \& Slangen, J.L. (1992). Visual stimulus change and the orienting reaction: event-related potential evidence for a two-stage process. Biological Psychology, 33, 97-114.

Koch, I., \& Philipp, A.M. (2005). Effects of response selection on the task repetition benefit in task switching. Memory \& Cognition, 33, 624-634

Lavric, A., Mizon, G., \& Monsell, S. (2008). Neurophysiological signature of effective anticipatory task-set control: a task-switching investigation. European Journal of Neuroscience, 28, 1016-1029

Lorist, M., Klein, M., Nieuwenhuis, S., De Jong, R., Mulder, G \& Meijman., T. (2000). Mental fatigue and task control: Planning and Preparation. Psychophysiology, 37, 614-625. 
Logan, G.D., \& Schneider, D.W. (2006) Interpreting Instructional Cues in Task Switching Procedures: The Role of Mediator Retrieval. Journal of Experimental Psychology: Learning, Memory, and Cognition, 32, 347-363.

Meiran, N. (2000). Reconfiguration of stimulus task sets and response task sets during task switching. In S. Monsell \& J. Driver (Eds.), Attention and Performance XVIII (pp. 377-399). Cambridge, MA: MIT Press.

Miniussi, C., Marzi, C.A., \& Nobre, A.C. (2005). Modulation of brain activity by selective task sets observed using event-related potentials. Neuropsychologia, 43, 1514-1528

Nicholson, R., Karayanidis, F., Poboka, D., Heathcote, A., \& Michie, P. (2005). Electrophysiological correlates of anticipatory task-switching processes. Psychophysiology, 42, 540-554.

Nicholson, R., Karayanidis, F., Bumak, E., Poboka, D., \& Michie, P.T. (2006a). ERPs dissociate the effects of switching task sets and task cues. Brain Research, 1095, 107-123

Nicholson, R., Karayanidis, F., Davies, A., \& Michie, P.T. (2006b). Components of task-set reconfiguration: Differential effects of 'switch-to' and 'switch-away' cues.

Nieuwenhuis, S., \& Monsell, S. (2002). Residual costs in task switching: testing the failure-to-engage hypothesis. Psychonomic Bulletin and Review, 9, 86-92

Poboka, D., Heathcote, A., Karayanidis, F., Nicholson, R., (2005). An Investigation of Task Switch Costs: Preparation Activation, Timing and Readiness Decay. International Conference on Attentional Control, Jan 4-7, National Chung Cheng University, Taiwan.

Provost, A., Brown, S., Heathcote, A.J., \& Karayanidis, F. (in preparation). A revised orthogonal polynomial trend analysis (OPTA) procedure for single-trial ERP waveform estimation across the RT distribution. 
Regan, D., (1989). Human brain electrophysiology: Evoked potentials and evoked magnetic fields in science and medicine. New York, Elsevier.

Rogers, R. D., \& Monsell, S. (1995). Costs of predictable switch between simple cognitive tasks. Journal of Experimental Psychology: General, 124, 207-231.

Rubinstein, J., Meyer, D. E., \& Evans, J. E. (2001). Executive control of cognitive processes. Journal of Experimental Psychology: Human Perception and Performance, 27, 763-797.

Ruge, H., Stoet, G.T., \& Naumann, E. (2006). Attentional set mixing: effects on target selection and selective response activation. Psychophysiology, 43, 413-421.

Rushworth, M. F. S., Passingham, R. E., \& Nobre, A. C. (2005). Components of attentional set-switching. Experimental Psychology, 52, 83-98

Semlitsch, H. V., Anderer, P., Schuster, P., \& Presslich, O. (1986). A solution for reliable and valid reduction of ocular artefacts. Psychophysiology, 23, 695-703

Shelley, A. M., Ward, P. B., Michie, P. T., Andrews, S., Mitchell, P. F., Catts, S. V., et al. (1991). The effect of repeated testing on ERP components during auditory selective attention. Psychophysiology, 28, 496-510

Spencer, K. (2004). Averaging, Detection, and Classification of Single-Trial ERPs. In T. Handy (Ed.), Event-Related potentials: A Methods handbook (pp.209-228). Cambridge, MA: MIT Press.

Swainson, R., Jackson, S.R. \& Jackson, G.M. (2006). Using advance information in dynamic cognitive control: An ERP study of task-switching. Brain Research, 1105, 61-72

Van der Lubbe, R., \& Woestenburg, J. (1997). Modulation of early ERP components with peripheral precues: a trend analysis. Biological Psychology, 45, 143-158.

Van der Lubbe, R., \& Woestenburg, J. (1999). The influence of peripheral precues on the tendency to react towards a lateral relevant stimulus with multiple-item arrays. Biological Psychology, 51, 1-21. 
Van der Lubbe, R., \& Woestenburg, J. (2000). Location selection in the visual domain. Psychophysiology, 37, 662-676.

Vasey, M. W., \& Thayer, J. F. (1987). The continuing problem of false positives in repeated measures ANOVA in psychophysiology: A multivariate solution. Psychophysiology, 24, 479-486

Woestenburg, J. C., Verbaten, M. N., Van Hees, H. H., \& Slangen, J. L. (1983). Single trial ERP estimation in the frequency domain using orthogonal polynomial trend analysis (OPTA): Estimation of individual habituation. Biological Psychology, 17, 173-191. 


\section{Figure Captions}

Figure 1. A. Mean RT at each of the twenty percentile ranks used in the analyses. B. Mean amplitude of the positivity averaged over midline and right parietal sites (300-400ms) and pre-target negativity averaged over midline and right central sites (500-600ms) across the range of the RT percentile ranks.

Figure 2. Cue-locked average waveforms for switch and repeat trials are presented here for ten trials that represent RT percentile ranks in $10 \%$ increments starting at the $5^{\text {th }}$ percentile. A. Difference waveforms derived by subtracting repeat trial from switch trial waveform at each RT percentile interval. B. ERP waveforms for repeat and switch trials. C. ERP waveforms superimposed for the $5^{\text {th }}$ and the $95^{\text {th }} \mathrm{RT}$ percentile. D. ERP waveforms superimposed for repeat and switch trials matched on RT ( 550-560ms).

Figure 3. Cue-locked ERP waveforms for one participant depicted at Pz for switch trials. No-OPTA data (left) represent conventional ERP averaging over the same trial numbers as the OPTA processed data (right). 


\section{Mean Amplitude}
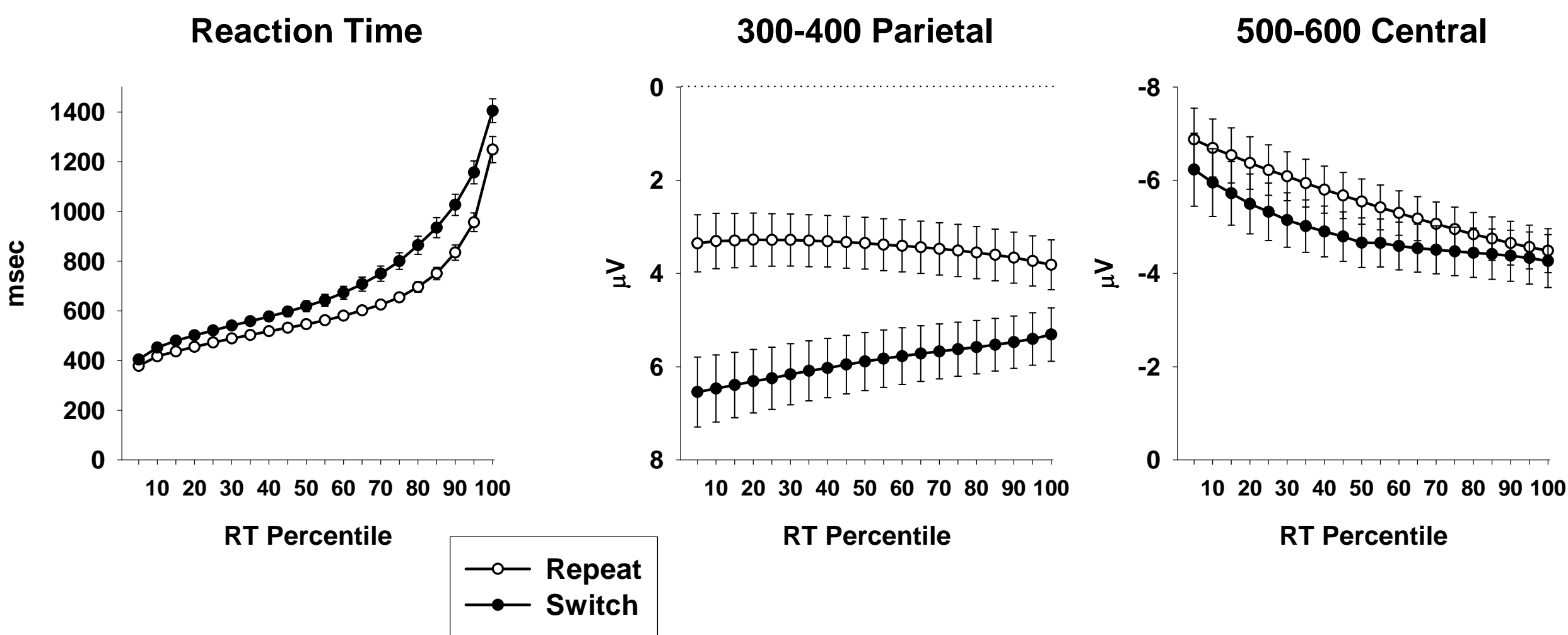


\section{A. (Switch - Repeat)}

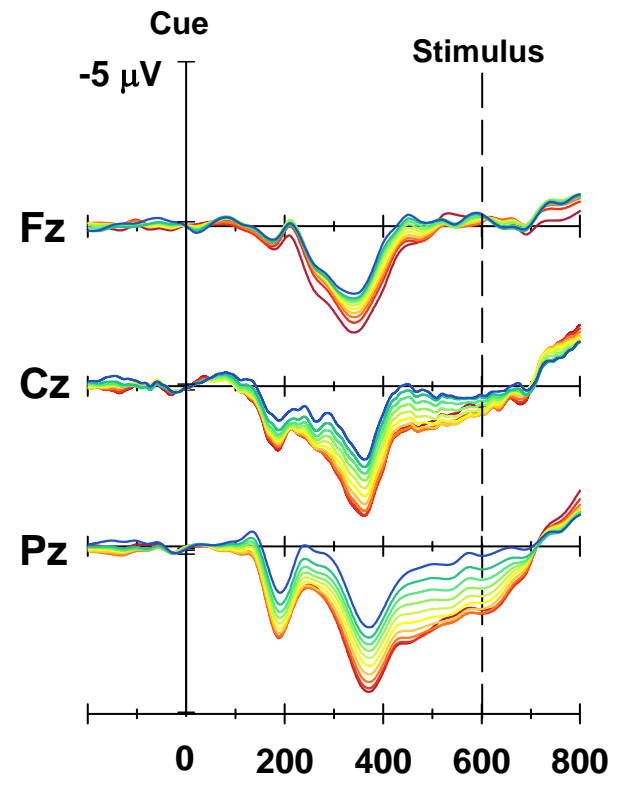

C. 5th vs 95th Percentile

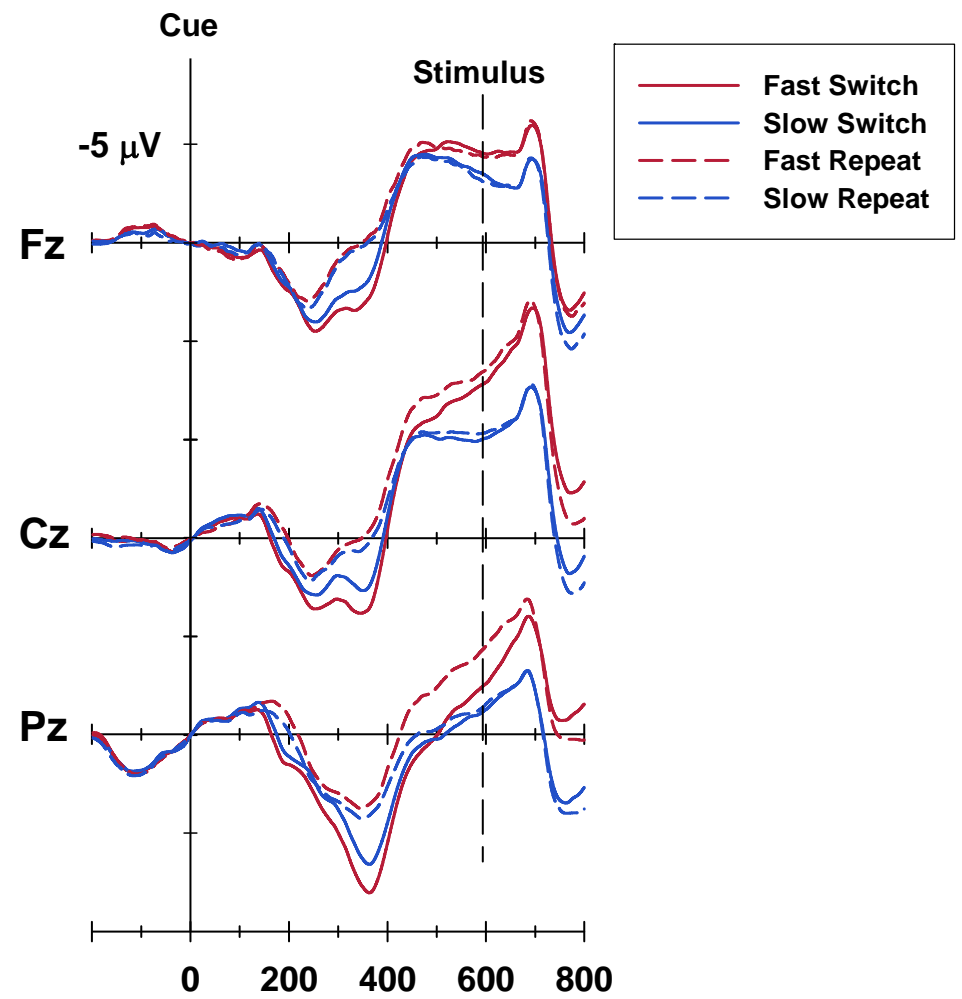

B. Repeat

Switch

Cue
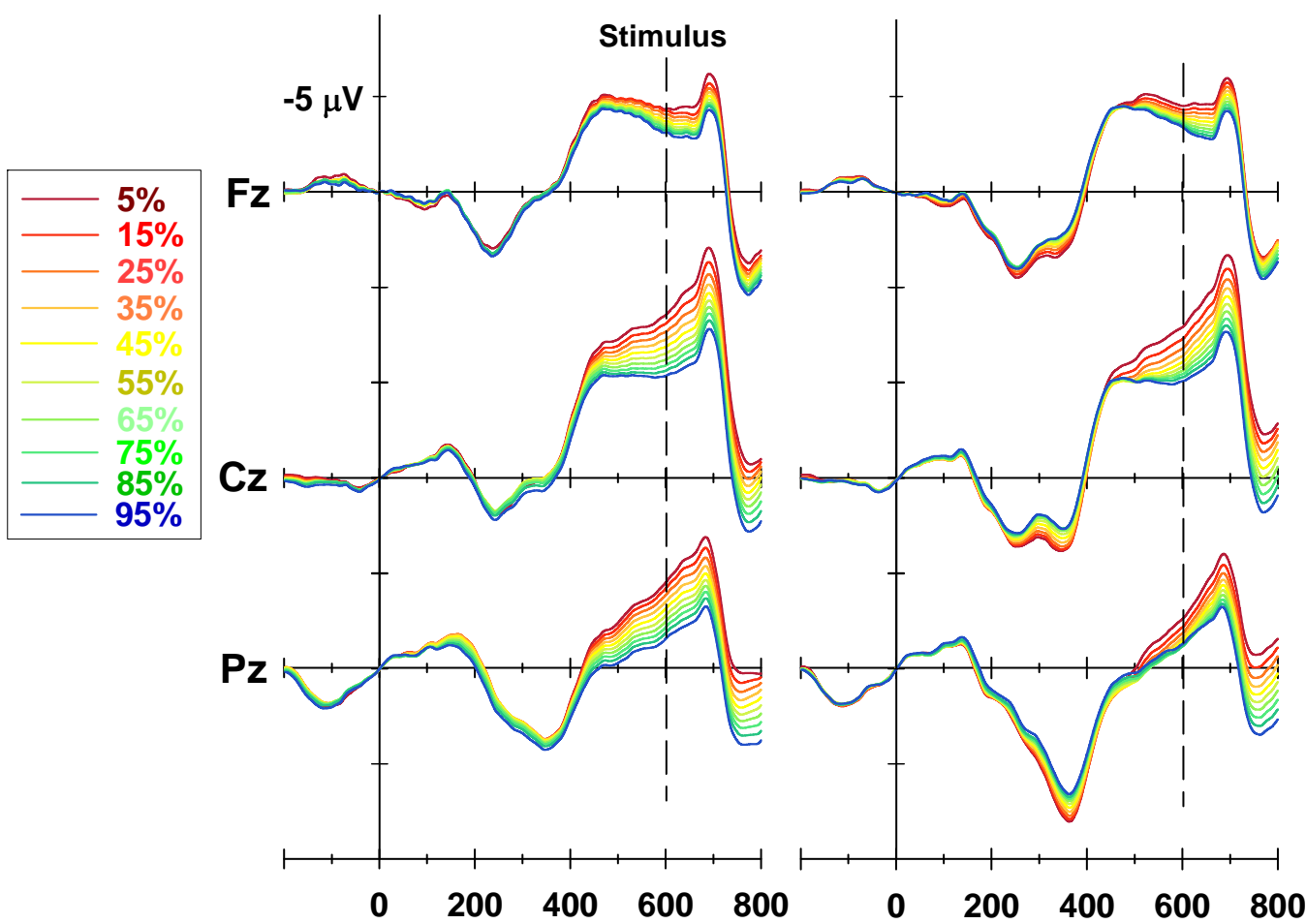

D. Switch and Repeat Equated for RT

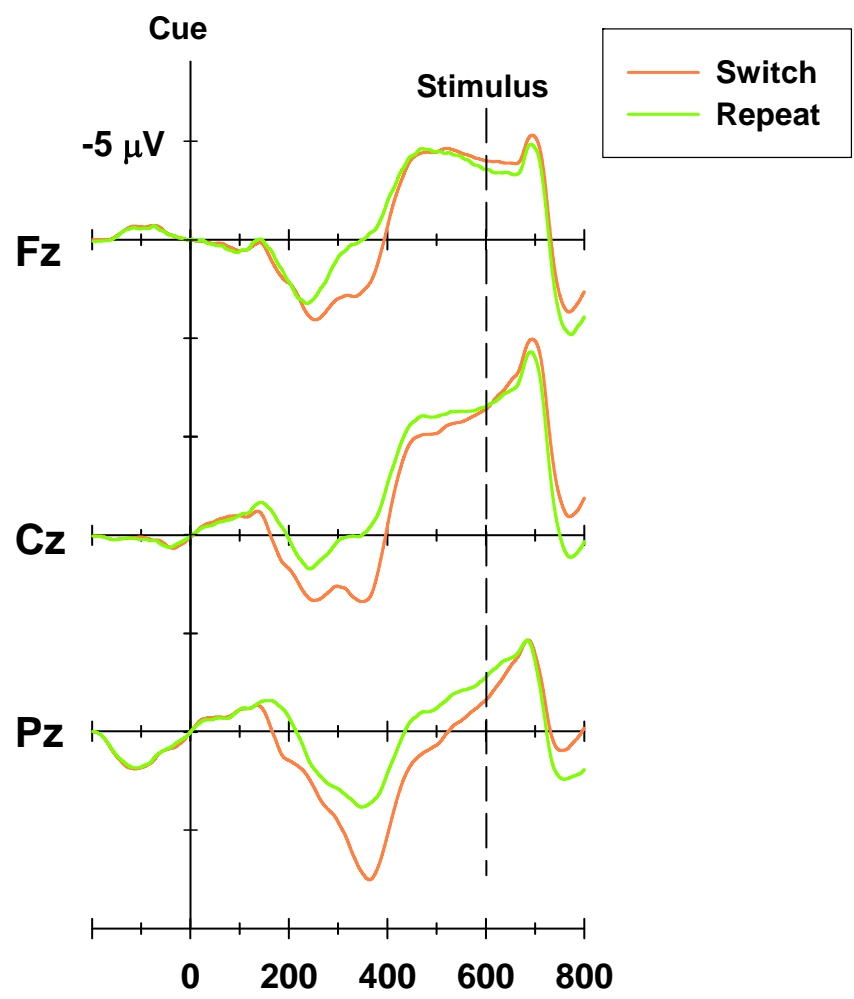


FIGURE 3
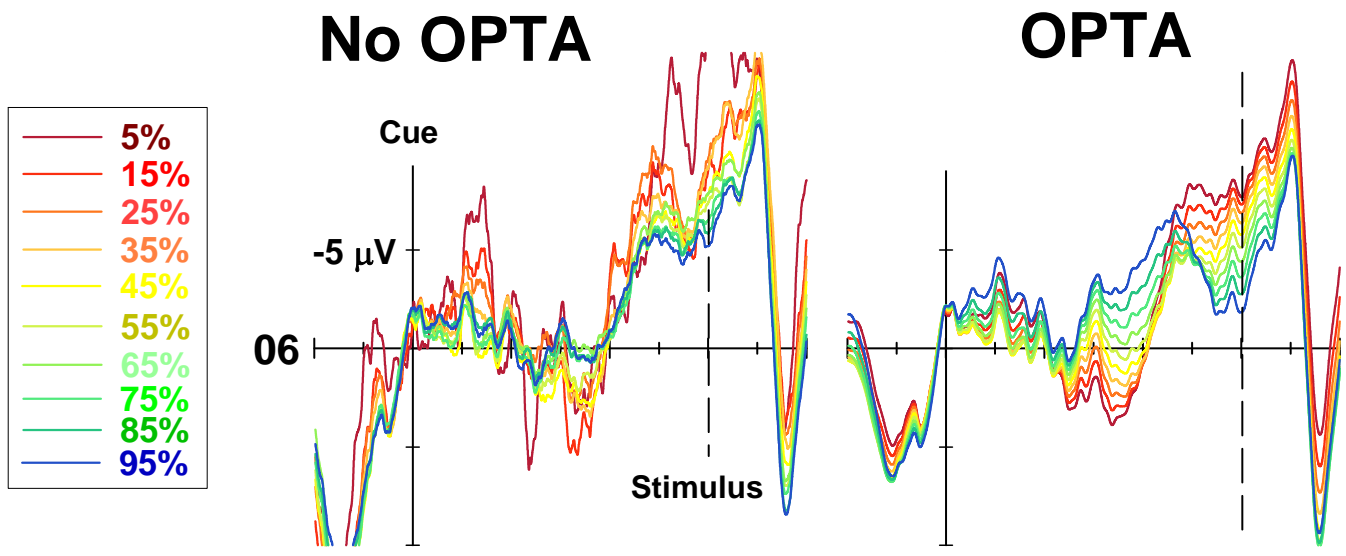